\title{
The insulator/Chern-insulator transition in the Haldane model
}

\author{
T. Thonhauser ${ }^{1}$ and David Vanderbilt ${ }^{1}$ \\ ${ }^{1}$ Department of Physics and Astronomy, Rutgers, \\ The State University of New Jersey, Piscataway, New Jersey 08854, USA.
}

(Dated: February 6, 2008)

\begin{abstract}
We study the behavior of several physical properties of the Haldane model as the system undergoes its transition from the normal-insulator to the Chern-insulator phase. We find that the density matrix has exponential decay in both insulating phases, while having a power-law decay, more characteristic of a metallic system, precisely at the phase boundary. The total spread of the maximally-localized Wannier functions is found to diverge in the Chern-insulator phase. However, its gauge-invariant part, related to the localization length of Resta and Sorella, is finite in both insulating phases and diverges as the phase boundary is approached. We also clarify how the usual algorithms for constructing Wannier functions break down as one crosses into the Chern-insulator region of the phase diagram.
\end{abstract}

PACS numbers: 73.43.-f, 73.20.At, 11.30.Rd

\section{INTRODUCTION}

The band structure of any insulator is characterized by a certain discrete topological index known as the Chern invariant $^{1}$ which encodes information about the phase evolution of the Bloch functions around the boundary of the Brillouin zone (see Sec. II). Insulators can thus be classified as "normal insulators" or "Chern insulators" depending on whether or not the Chern invariant vanishes. The latter case requires breaking of time-reversal symmetry, so insulating ferromagnets and ferrimagnets could be candidates for Chern insulators. While models for Chern insulators can be constructed theoretically, ${ }^{2}$ no experimental realizations are yet known to occur in nature. A Chern insulator, if found to exist, would have the remarkable feature of showing a quantum Hall effect in the absence of a macroscopic magnetic field. Hence, Chern insulators may also be referred to as "quantum Hall insulators."

Although the basic theory of Chern insulators was formulated in the 1980's, not much is known theoretically about the general features of the electronic band structure of such insulators. In the last 15 years or so, the theory of normal insulators has been greatly enriched by a deeper understanding of electric polarization, ${ }^{3}$ orbital magnetization, ${ }^{4,5}$ linear-scaling theory, methods for constructing Wannier functions, ${ }^{6}$ the spatial decay of Wannier functions and of the one-particle density matrix, ${ }^{7}$ and related measures of localization. ${ }^{8-10}$ However, all of this work implicitly assumed the presence of time-reversal symmetry, and thus was limited to the case of normal insulators. It is therefore of considerable interest to revisit many of these same issues, and to reconsider whether, or how, the previous conclusions generalize to the case of Chern insulators. For example, what are the decay properties of the one-particle density matrix in a Chern insulator? Can Wannier functions be constructed, and if not, in what way do the usual construction procedures fail? If one inspects closely related measures of localization such as the gauge-invariant part of the Wannier spread functional, ${ }^{6}$ the localization length of Resta and Sorella, ${ }^{8}$ or the second-cumulant moment of the electron distribution, ${ }^{9,10}$ does the localization remain finite in a Chern insulator, or does it diverge?

Furthermore, an intriguing feature of Chern-insulating systems is that a phase boundary separating the Chern insulator from a normal insulator may occur. Such a normal-insulator/Chern-insulator (NI/CI) transition is an example of a class of topological transitions that have become of considerable current interest, at which a topological invariant changes discontinuously across a phase boundary. ${ }^{11}$ Such transitions normally appear within the theory of correlated states. ${ }^{12,13}$ The NI/CI transition, on the other hand, occurs in a non-interacting context, and can therefore be studied at a level of detail, and tested with numerical calculations, in a way that is difficult for correlated models. In addition, Chern insulators are closely related to so-called "spin Hall insulators," which have also been the subject of a recent surge of interest. ${ }^{14}$ Thus, there is a special opportunity associated with the study of this particular topological insulator/insulator transition.

In this paper, we investigate several aspects of the electronic structure near the NI/CI transition in the twodimensional Haldane model. ${ }^{2}$ We choose the Haldane model because it is one of the simplest models that exhibits a quantum-Hall insulator state. The underlying idea of this model is to break time-reversal symmetry so that the transverse conductivity $\sigma_{x y}$, which is odd under time-reversal, can become nonzero. Usually, the quantum Hall effect is associated with a gap at the Fermi level resulting from a splitting of the spectrum into Landau levels by a macroscopic magnetic field. In the Haldane model, however, there is a degeneracy between the valence and conduction bands at certain high-symmetry k-points when both inversion and time-reversal symmetry are present. If a gap is opened by the breaking of inversion symmetry, the system becomes a normal insulator. However, if the gap opens as a result of breaking time-reversal symmetry, the system turns into a Chern 
insulator.

We have organized this paper as follows. In Sec. II we introduce the Chern invariant, which will henceforth be used to classify the state of our system. The basics of the Haldane model are reviewed in Sec. III. Thereafter, we focus on the problems occurring when constructing Wannier functions for Chern insulators (Sec. IV), the behavior of the spread functional (Sec. V), and the decay of the density matrix (Sec. VI) as the system transitions from the normal insulator phase into the Chern-insulator phase. We conclude and give an outlook in Sec. VII.

\section{THE CHERN INVARIANT}

We restrict ourselves to the case of a one-particle Hamiltonian $H$ having Bloch eigenvalues $\epsilon_{n \mathbf{k}}$ and eigenstates $\left|\psi_{n \mathbf{k}}\right\rangle$. The cell-periodic part of the Bloch function $u_{n \mathbf{k}}(\mathbf{r})=e^{-i \mathbf{k} \cdot \mathbf{r}} \psi_{n \mathbf{k}}(\mathbf{r})$ is then an eigenfunction of the effective Hamiltonian $H(\mathbf{k})=e^{-i \mathbf{k} \cdot \mathbf{r}} H e^{i \mathbf{k} \cdot \mathbf{r}}$. We consider electrons to be spinless, but factors of two can easily be inserted for non-interacting spin channels.

We can now define the Chern invariant ${ }^{1}$ for an insulator, defined here as a system with a gap in the singleparticle density of states separating occupied and unoccupied states, to be

$$
\mathbf{C}=\frac{i}{2 \pi} \int_{\mathrm{BZ}} d \mathbf{k} \sum_{n}^{\mathrm{occ}}\left\langle\partial_{\mathbf{k}} u_{n \mathbf{k}}|\times| \partial_{\mathbf{k}} u_{n \mathbf{k}}\right\rangle,
$$

where $\mathrm{BZ}$ denotes an integral over the Brillouin zone and $\partial_{\mathbf{k}}=\partial / \partial \mathbf{k}$. The cross product notation in Eq. (1) implies, for example, that $C_{z}$ contains terms involving $\left\langle\partial_{k_{x}} u_{n \mathbf{k}} \mid \partial_{k_{y}} u_{n \mathbf{k}}\right\rangle-\left\langle\partial_{k_{y}} u_{n \mathbf{k}} \mid \partial_{k_{x}} u_{n \mathbf{k}}\right\rangle$. For non-interacting electrons, ${ }^{15,16}$ the Chern invariant is quantized in units of reciprocal-lattice vectors G. For the case of a twodimensional system with only a single occupied band, Eq. (1) becomes

$$
C=\frac{i}{2 \pi} \int_{\mathrm{BZ}} d \mathbf{k}\left\langle\partial_{\mathbf{k}} u_{\mathbf{k}}|\times| \partial_{\mathbf{k}} u_{\mathbf{k}}\right\rangle .
$$

In two dimensions the Chern invariant is a pseudo-scalar called the Chern number which can only take integer values. Alternatively, we can write the Chern number in terms of the Berry connection $\mathbf{A}(\mathbf{k})=i\left\langle u_{\mathbf{k}}\left|\partial_{\mathbf{k}}\right| u_{\mathbf{k}}\right\rangle$ and the Berry curvature $\Omega(\mathbf{k})=\nabla_{\mathbf{k}} \times \mathbf{A}(\mathbf{k})$ as

$$
C=\frac{1}{2 \pi} \int_{\mathrm{BZ}} d \mathbf{k} \Omega(\mathbf{k})=\frac{1}{2 \pi} \oint_{\mathrm{BZ}} d \mathbf{k} \cdot \mathbf{A}(\mathbf{k}) .
$$

A Chern insulator is now simply defined as an insulator with a nonzero Chern invariant. Conversely, we define a normal insulator to be an insulator with zero Chern invariant. Hence, the NI/CI transition is characterized by a change of the Chern invariant from zero to a nonzero value.

The Chern invariant of Eqs. (1) and (2) is gauge invariant,${ }^{5}$ i.e., invariant with respect to the choice of

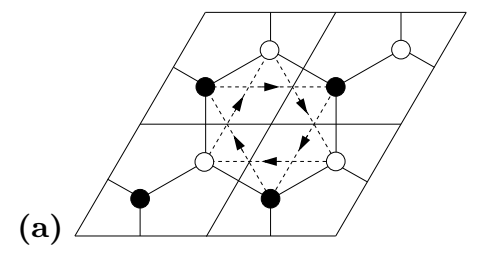

(b)

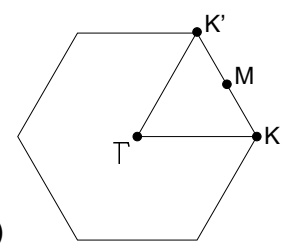

FIG. 1: (a) Four unit cells of the Haldane model. Filled (open) circles have site energy $-\Delta(+\Delta)$. The first-nearest neighbor hopping $t_{1}$ is real, while the second-nearest neighbor hopping $t_{2} e^{i \varphi}$ has a complex phase. Arrows indicate the direction of positive phase hopping. The Wigner-Seitz unit cell corresponds to the hexagon in the center of the plot. (b) First Brillouin zone of the Haldane model with high-symmetry points marked.

phases of the $\left|u_{n \mathbf{k}}\right\rangle$, or in the more general multiband case, the choice of unitary rotations applied to transform the occupied states among themselves at a given $\mathbf{k}$. It can be shown that in normal insulators it is always possible to make a gauge choice such that the Bloch orbitals are periodic in k-space (i.e., $\left|\psi_{n \mathbf{k}+\mathbf{G}}\right\rangle=\left|\psi_{n \mathbf{k}}\right\rangle$ ) and smooth in $\mathbf{k}$ (i.e., continuous and differentiable), whereas no such choice is possible for a Chern insulator. ${ }^{17}$

\section{THE HALDANE MODEL}

Here we provide a brief review of Haldane's model and its properties, as discussed in detail in Ref. [2]. As illustrated in Fig. 1, the Haldane model is comprised of a honeycomb lattice having two tight-binding sites per cell with site energies $\pm \Delta$, a real first-neighbor hopping $t_{1}$, and a complex second-neighbor hopping $t_{2} e^{ \pm i \varphi}$. The model can also be thought of as consisting of two sublattices $A$ and $B$ corresponding to the sites with energies $+\Delta$ and $-\Delta$, respectively. Note that the macroscopic magnetic flux through the unit cell is indeed zero, resulting in a vanishing macroscopic magnetic field. This follows directly from the fact that the first-nearest neighbor hopping is real and no phase is picked up when hopping around the Wigner-Seitz unit cell. This, however, does not rule out a microscopic magnetic field that averages to zero over the unit cell. Note that the wavevector $\mathbf{k}$ is still a good quantum number under these conditions.

Let $\mathbf{a}_{1}, \mathbf{a}_{2}$, and $\mathbf{a}_{3}$ be the vectors pointing from a site of the $B$ sublattice to its three nearest $A$ neighbors, such that $\hat{\mathbf{z}} \cdot \mathbf{a}_{1} \times \mathbf{a}_{2}>0$ and $\hat{\mathbf{x}} \cdot \mathbf{a}_{1}>0$. If we furthermore define the vectors $\mathbf{b}_{1}=\mathbf{a}_{2}-\mathbf{a}_{3}, \mathbf{b}_{2}=\mathbf{a}_{3}-\mathbf{a}_{1}$, and $\mathbf{b}_{3}=\mathbf{a}_{1}-\mathbf{a}_{2}$, then the Hamiltonian of the Haldane model 


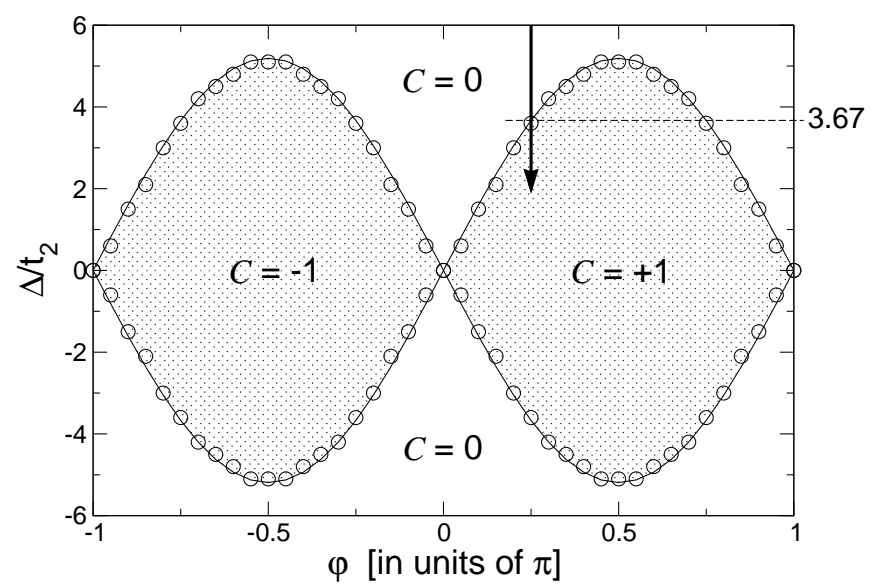

FIG. 2: Chern number of the bottom band of the Haldane model as a function of the parameters $\varphi$ and $\Delta / t_{2}\left(t_{1}=1\right.$, $\left.t_{2}=1 / 3\right)$. Analytic results are plotted as solid sinusoidal lines, whereas numerical results are depicted by circles. The vertical line shows the range of parameters that we have chosen for all our calculations.

can be written as

$$
\begin{aligned}
& H(\mathbf{k})=\mathbf{I} 2 t_{2} \cos \varphi \sum_{i} \cos \left(\mathbf{k} \cdot \mathbf{b}_{i}\right) \\
& +\boldsymbol{\sigma}_{1} t_{1} \sum_{i} \cos \left(\mathbf{k} \cdot \mathbf{a}_{i}\right)+\boldsymbol{\sigma}_{2} t_{1} \sum_{i} \sin \left(\mathbf{k} \cdot \mathbf{a}_{i}\right) \\
& +\boldsymbol{\sigma}_{3}\left(\Delta-2 t_{2} \sin \varphi \sum_{i} \sin \left(\mathbf{k} \cdot \mathbf{b}_{i}\right)\right)
\end{aligned}
$$

where the $\boldsymbol{\sigma}_{i}$ are the Pauli matrices and $\mathbf{I}$ is the identity matrix.

The Chern number can now be calculated analytically or numerically according to Eq. (2) or (3). For our tests, we have chosen a lattice constant equal to unity, $t_{1}=1$, and $t_{2}=1 / 3$. If the Chern number of the bottom band is mapped out as a function of the remaining model parameters $\varphi$ and $\Delta / t_{2}$, we obtain the Haldane phase diagram shown in Fig. 2. Since we are interested in studying the transition from a normal insulator to a Chern insulator, we choose for all our calculations below a path in the phase diagram that crosses the phase boundary. Specifically, we traverse the vertical line in Fig. 2 where the phase $\varphi$ is fixed at $\pi / 4$ and $\Delta / t_{2}$ is reduced from 6 to 2 . At the critical value $\left(\Delta / t_{2}\right)_{\mathrm{cr}}=3 \sqrt{3} \sin (\pi / 4) \approx 3.67$, the phase boundary is crossed.

The band structure of the Haldane model is plotted in Fig. 3 along some high-symmetry lines in the Brillouin zone (see Fig. 1b). It shows a remarkable feature as the system passes through $\left(\Delta / t_{2}\right)_{\mathrm{cr}}$. In the normal-insulator region, the two bands are separated by a finite gap. As the critical value is approached, the gap at $K$ gets smaller and smaller. Finally, exactly at $\left(\Delta / t_{2}\right)_{\mathrm{cr}}$ the bands touch at $K$ in such a way that the dispersion relation is linear. Such points are also referred to as Dirac points. When going further into the Chern-insulator region, the bands

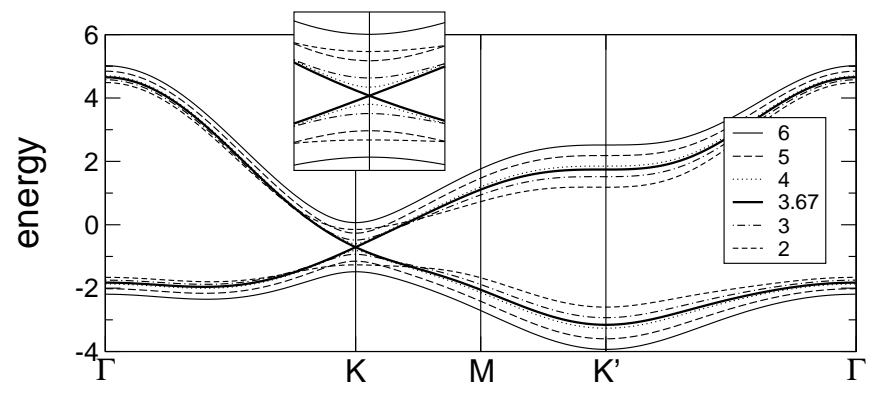

FIG. 3: Band structure of the Haldane model along some high-symmetry lines for several values of $\Delta / t_{2}$ along the path marked in Fig 2. The inset shows a magnification of the bands at $K$. Note that at $\left(\Delta / t_{2}\right)_{\mathrm{cr}}$ the dispersion is linear.

separate again. Note that our specific choice of $t_{1}=1$ and $t_{2}=1 / 3$ prevents the bands from overlapping. If $\Delta$ and $t_{2} \sin \varphi$ are both chosen to be zero, two Dirac points form at $K$ and $K^{\prime}$, and the Haldane model then becomes an appropriate model for a graphene sheet. ${ }^{18}$

In the normal-insulator region of the Haldane model the Chern number of each band is zero, so that the total Chern number (the sum of the Chern numbers of the upper and lower bands) is obviously also zero. When the phase boundary is crossed, the Chern numbers of the upper and lower bands become \pm 1 , but their sum still remains zero. The closure and reopening of the gap as the NI/CI boundary is crossed corresponds to the "donation" of a Chern unit from one band to another through the temporarily formed Dirac point. In the present case, the total Chern number must always remain zero because the model, having a tight-binding form, assumes Wannier representability of the overall band space, and a non-zero Chern number is inconsistent with such an assumption. More generally, the total Chern number of a group of bands should not change when a gap closure and reopening occurs among the bands of the group, as long as the gaps between this group and any lower or higher bands remains open.

It is possible to argue on very general grounds that a finite sample cut from a Chern insulator must have conductive channels, otherwise known as chiral edge states, that circulate around the perimeter of the sample ${ }^{19}$ in much the same way as for the quantum Hall effect. ${ }^{20,21} \mathrm{It}$ is therefore of interest to investigate the electronic structure of the Haldane model from the point of view of the surface band structure. We consider a sample that is finite in the $\mathbf{b}_{3}$ direction (specifically, 30 cells wide) and has periodic boundary conditions along the $\mathbf{b}_{2}$ direction (the $\mathbf{b}_{i}$ are defined above Eq. (4)); its states can be labeled by a wavevector $k_{y}$ running from $-\pi / a$ to $+\pi / a$, where $a$ is the repeat unit in the $y$ direction. The energy eigenvalues are plotted vs. $k_{y}$ for several values of $\Delta / t_{2}$ in Fig. 4. At first sight, the surface band structure shows qualitatively the same information as the bulk band structure in Fig. 3. For $\Delta / t_{2}=6$, the valence and 


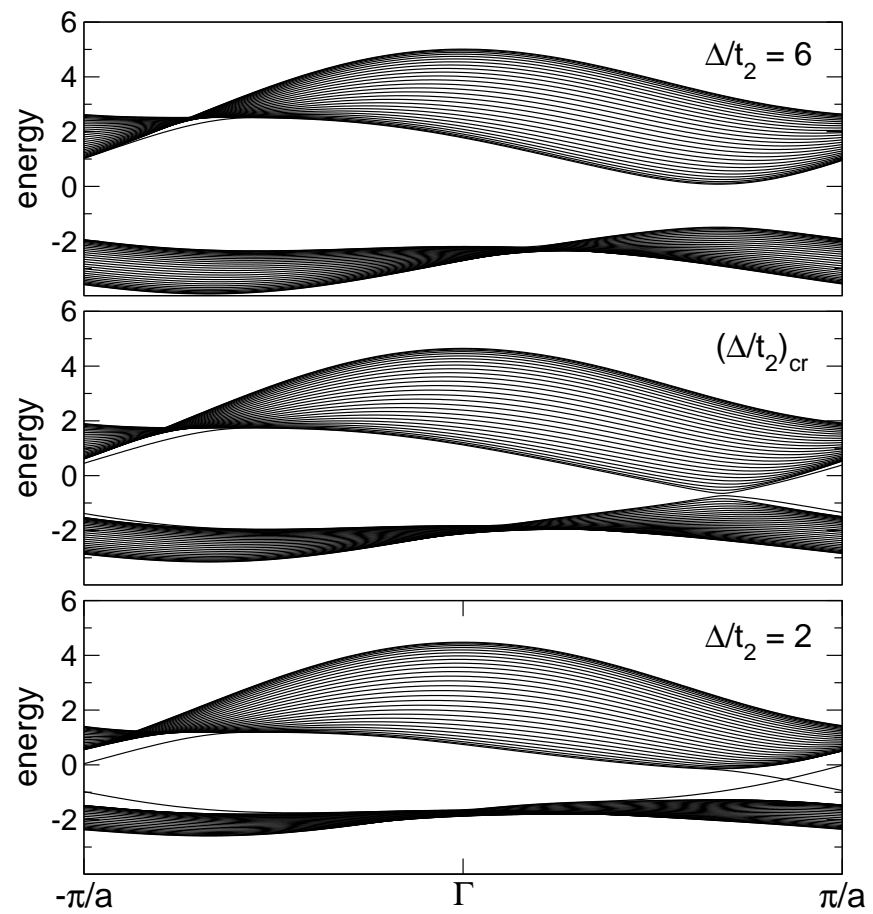

FIG. 4: Energy vs. wavevector $k_{y}$ for the Haldane model in a strip geometry 30 cells wide along $\mathbf{b}_{3}$ direction and extending infinitely along $\mathbf{b}_{2}$ direction. For $\Delta / t_{2}<\left(\Delta / t_{2}\right)_{\mathrm{cr}}$ (bottom panel), chiral edge states are visible.

conduction bands are separated by a finite gap. At the Chern transition a Dirac point forms, showing the characteristic linear dispersion expected around such a point. However, when we go deeper into the Chern insulator, the surface band structure reveals a new behavior: one surface band now crosses from the lower manifold to the upper one with increasing $k_{y}$, and another crosses in the opposite direction. Further inspection shows that the upgoing and downgoing states are localized to the right and left surfaces of the strip, respectively. Thus, if the Fermi level lies in the bulk gap, there will be metallic states with Fermi velocities parallel to the surfaces and with opposite orientation, i.e., a chiral (counterclockwise) circulation of edge states around the perimeter of the sample, as expected.

\section{BREAKDOWN OF WANNIER-FUNCTION CONSTRUCTION AT THE CHERN TRANSITION}

We now study aspects of the NI/CI transition that are related to Wannier functions (WFs) and electron localization. We expect that in the normal-insulator phase, it should be straightforward to construct Wannier functions via a k-space construction. The term "Wannier function" is usually applied only in the case of periodic systems, but for finite samples one can construct well localized Boys orbitals $^{22}$ which play the same role and which map onto the WFs in the thermodynamic limit $n \rightarrow \infty$. Thus, if we cut a finite sample from a normal-insulator realization of the Haldane model, we also expect it to be straightforward to construct such Boys orbitals. The question then arises as to what, precisely, will "go wrong" with these procedures if one tries to do the same on the Cherninsulator side of the transition. In particular, for a finite sample cut from the Haldane model, it is unclear how the system would "know" whether the finite sample corresponds to the normal-insulator or Chern-insulator side of the transition, and how the construction would break down in the latter case. In this Section, we investigate these issues, first in the context of the real-space construction, and then later from the $\mathbf{k}$-space point of view.

We start, then, by considering finite $n \times n$ samples of the Haldane model. We can interpret Fig. 1 as showing a picture of a finite sample of size $n=2$; we study similarly-constructed samples of size $n=10,20,30$, and 40. For each sample, the Boys orbitals are constructed as follows. We define the projection operator onto the occupied states as

$$
P=\sum_{n}^{\text {occ }}\left|\psi_{n}\right\rangle\left\langle\psi_{n}\right|,
$$

and we choose a set of well-localized "trial" orbitals $\left|t_{\alpha}\right\rangle$, equal in number to the number of occupied states, that we want the Boys orbitals to be roughly modeled after. We then construct the projected trial functions $\left|y_{\alpha}\right\rangle=P\left|t_{\alpha}\right\rangle$. Since $\rho\left(\mathbf{r}, \mathbf{r}^{\prime}\right)=\left\langle\mathbf{r}|P| \mathbf{r}^{\prime}\right\rangle$ is expected to decay exponentially in $\left|\mathbf{r}-\mathbf{r}^{\prime}\right|$ for an insulator (see Sec. VI), we expect the $\left|y_{\alpha}\right\rangle$ to be localized as well, and as long as they are not overcomplete they will span the occupied space of interest. However, they are not orthonormal, so the last step is to carry out a symmetric orthonormalization. ${ }^{23}$ This is done by computing the overlap matrix

$$
S_{\alpha \beta}=\left\langle y_{\alpha} \mid y_{\beta}\right\rangle
$$

and then constructing the final Boys orbitals $\left|\omega_{\alpha}\right\rangle$ as

$$
\left|w_{\alpha}\right\rangle=\sum_{\beta}\left(S^{-1 / 2}\right)_{\beta \alpha}\left|y_{\beta}\right\rangle .
$$

In the context of the Haldane model, it is natural to choose the trial functions to be a set of $\delta$-functions located on the sites of the lower-energy sublattice. With this choice, we can now study the lowest and highest eigenvalue of $S_{\alpha \beta}$ as the parameter $\Delta / t_{2}$ traverses the path shown in Fig. 2. While the highest eigenvalue remains very close to 1 , the lowest eigenvalue drops and rapidly approaches zero in the Chern-insulator region, i.e., for $\Delta / t_{2}$ values below the critical value of $\left(\Delta / t_{2}\right)_{\mathrm{cr}} \approx 3.67$, as shown in Fig. 5 . The slope of the "drop" depends on the size of the sample and becomes steeper as the sample size gets larger. For any given value of $\Delta / t_{2}<\left(\Delta / t_{2}\right)_{\mathrm{cr}}$, the lowest eigenvalue appears 


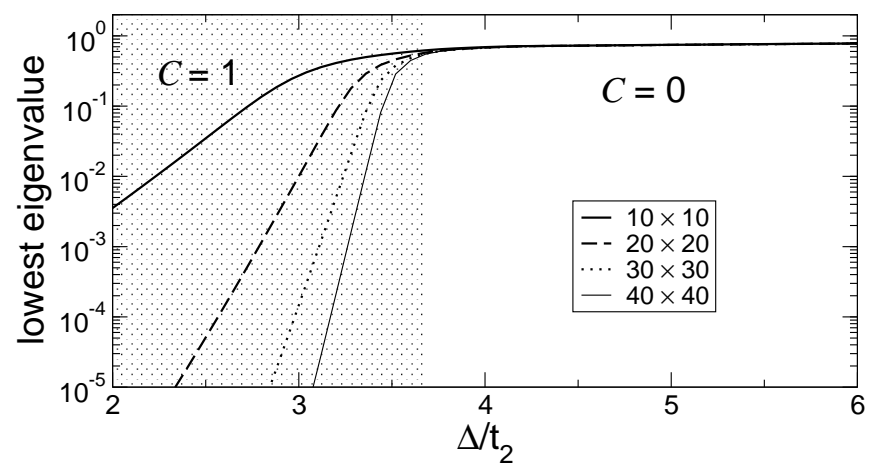

FIG. 5: Lowest eigenvalue of the overlap matrix $S_{\alpha \beta}=$ $\left\langle y_{\alpha} \mid y_{\beta}\right\rangle$ as a function of the parameter $\Delta / t_{2}$ for different sample sizes $n \times n$. Note the logarithmic scale.

to approach zero exponentially with sample size. When the eigenvalue becomes too small, the inversion to obtain $S^{-1 / 2}$ becomes ill-conditioned, and the symmetric orthonormalization in Eq. (7) can no longer be carried out. It follows that Boys orbitals cannot be constructed in the Chern-insulator phase, at least not using this approach.

We now change perspective and look at the problem from the $\mathbf{k}$-space point of view, where we find that something similar happens. WFs for periodic samples are defined by

$$
|\mathbf{R} n\rangle=\frac{\Omega}{(2 \pi)^{3}} \int_{\mathrm{BZ}} d \mathbf{k} e^{-i \mathbf{k} \cdot \mathbf{R}}\left|\psi_{n \mathbf{k}}\right\rangle,
$$

where the inverse relation is

$$
\left|\psi_{n \mathbf{k}}\right\rangle=\sum_{\mathbf{R}} e^{i \mathbf{k} \cdot \mathbf{R}}|\mathbf{R} n\rangle
$$

In this notation $|\mathbf{R} n\rangle$ refers to the $n$ 'th WF in cell $\mathbf{R}$.

As mentioned previously, for systems with zero Chern invariant, the Bloch orbitals can always be chosen to obey a smooth and periodic gauge $\left|\psi_{n \mathbf{k}+\mathbf{G}}\right\rangle=\left|\psi_{n \mathbf{k}}\right\rangle$. However, if the Chern invariant becomes nonzero, this choice is no longer possible. ${ }^{17} \mathrm{In}$ this case it is possible to make a periodic gauge choice that is smooth almost everywhere, but there must be singularities ("vortices") somewhere in the interior of the BZ. For example, in two dimensions, assume a gauge choice that is periodic and also smoothly defined everywhere in the BZ except in a small disk located somewhere in the interior of the BZ. The periodic gauge choice implies that $\oint d \mathbf{k} \cdot \mathbf{A}(\mathbf{k})$ around the perimeter of the BZ must vanish. Applying Stoke's theorem as in Eq. (3), but now to the region excluding the small disk, implies that $\oint d \mathbf{k} \cdot \mathbf{A}(\mathbf{k})$ around the circumference of the small disk must approach $-C$ in the limit that the disk becomes small. For the Chern phase $(C \neq 0)$, this implies that there must be a vortex singularity in the phase choice inside the disk. If one attempts to construct WFs naively using Eq. (8), one then finds that the discontinuity in the phase choice of $\left|u_{n \mathbf{k}}\right\rangle$ at the vortex in $\mathbf{k}$-space

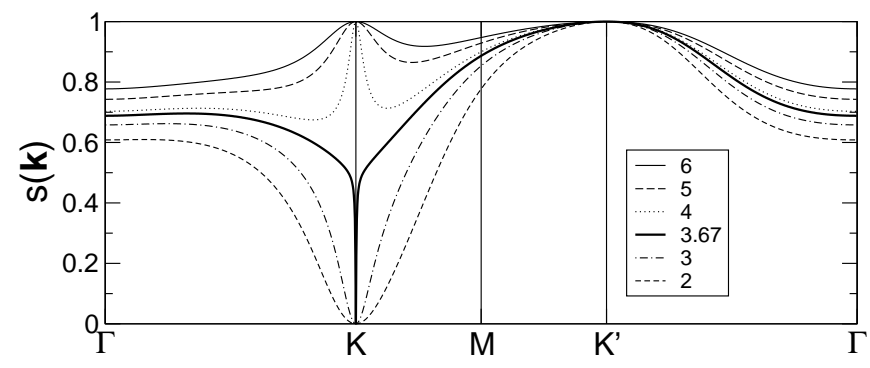

FIG. 6: Plot of $s(\mathbf{k})$ of Eq. (12) along some high-symmetry lines for several values of $\Delta / t_{2}$.

leads to the destruction of exponential localization of the WFs in real space.

We have investigated how this problem manifests itself if one attempts to construct WFs using standard k-space methods. Similar to the approach described in Ref. [6], we again adopt a projection method in which one chooses trial Bloch-like functions $\left|t_{\mathbf{k}}\right\rangle$ that are smooth and periodic in $\mathbf{k}$-space. This can be done by constructing the $\left|t_{\mathbf{k}}\right\rangle$ from a set of real-space trial functions $\left|t_{\alpha}\right\rangle$, i.e., $t_{\mathbf{k}}(\mathbf{r})=\sum_{\mathbf{R}} e^{i \mathbf{k} \cdot \mathbf{R}} t_{\alpha}(\mathbf{r}-\mathbf{R})$. Then one can construct projected states $\left|y_{\mathbf{k}}\right\rangle$ via

$$
\left|y_{\mathbf{k}}\right\rangle=P\left|t_{\mathbf{k}}\right\rangle=\sum_{\mathbf{k}^{\prime}}\left|\psi_{\mathbf{k}^{\prime}}\right\rangle\left\langle\psi_{\mathbf{k}^{\prime}} \mid t_{\mathbf{k}}\right\rangle=\left|\psi_{\mathbf{k}}\right\rangle\left\langle\psi_{\mathbf{k}} \mid t_{\mathbf{k}}\right\rangle
$$

and orthonormalized projected states

$$
\left|w_{\mathbf{k}}\right\rangle=s(\mathbf{k})^{-1 / 2}\left|y_{\mathbf{k}}\right\rangle
$$

where

$$
s(\mathbf{k})=\left\langle y_{\mathbf{k}} \mid y_{\mathbf{k}}\right\rangle=\left|\left\langle t_{\mathbf{k}} \mid y_{\mathbf{k}}\right\rangle\right|^{2}
$$

The WFs are then constructed by Fourier transforming to real space using Eq. (8) with $\left|w_{\mathbf{k}}\right\rangle$ substituted for $\left|\psi_{\mathbf{k}}\right\rangle$. Clearly, if $s(\mathbf{k})$ should vanish at some $\mathbf{k}$, this procedure would fail.

We can now study what happens if this construction procedure is applied to the Haldane model. We again use trial functions that are $\delta$-functions located on the lowerenergy sites. We study the behavior of $s(\mathbf{k})$ as a function of $\mathbf{k}$ throughout the BZ, while varying $\Delta / t_{2}$ along the line in Fig. 2. Results for $s(\mathbf{k})$ are plotted along some high-symmetry lines in Fig. 6. In the normal-insulator region, we find $0<s(\mathbf{k}) \leq 1$ for all $\mathbf{k}$. After the phase boundary has been crossed at $\left(\Delta / t_{2}\right)_{\mathrm{cr}}$, we find that there is one point $\mathbf{k}_{a}$ in the BZ for which $s\left(\mathbf{k}_{a}\right)=0$. There is also one point $\mathbf{k}_{b}$ for which $s\left(\mathbf{k}_{b}\right)=1$ exactly. In our numerical calculations, the locations of $\mathbf{k}_{a}$ and $\mathbf{k}_{b}$ coincide with the points $K$ and $K^{\prime}$ respectively. By experimenting with different trial functions, we have found that the precise locations of the minimum and maximum may deviate from $K$ and $K^{\prime}$, and the value at the maximum may be less than unity. However, we always find a point $\mathbf{k}_{a}$ at which $s\left(\mathbf{k}_{a}\right)=0$. This is the point at which $\left\langle\psi_{\mathbf{k}} \mid t_{\mathbf{k}}\right\rangle=0$; the robustness of such a zero-crossing can be understood 


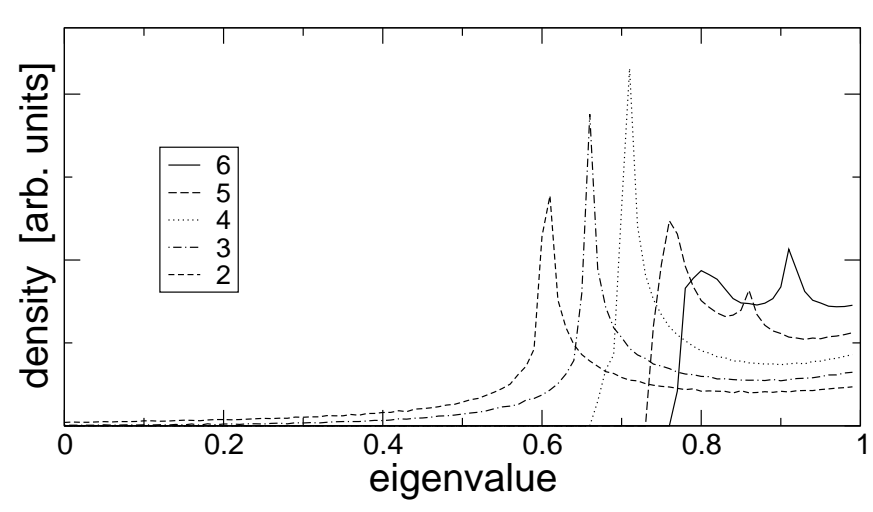

FIG. 7: Density of $s(\mathbf{k})$ values as a function of $\Delta / t_{2}$ obtained for periodic samples with a $300 \times 300 \mathbf{k}$-mesh.

heuristically by realizing that by adjusting the two parameters $k_{x}$ and $k_{y}$, the real and imaginary parts of the complex scalar $\left\langle\psi_{\mathbf{k}} \mid t_{\mathbf{k}}\right\rangle$ can generically both be made to vanish. From Eqs. (10-12) it follows that the phase of $\left|y_{\mathbf{k}}\right\rangle$ evolves by $2 \pi$ as one circles around $\mathbf{k}_{a}$, so that a vortex-like singularity is generated in the phase of $\left|w_{\mathbf{k}}\right\rangle$ about $\mathbf{k}_{a}$, with $\left|w_{\mathbf{k}}\right\rangle$ becoming ill-defined precisely at $\mathbf{k}_{a}$. Thus, the construction of well-localized WFs is no longer possible.

Instead of focusing only on the lowest eigenvalue, we plot in Fig. 7 the "density of overlap values" $s(\mathbf{k})$. In the normal-insulator region of $\Delta / t_{2}$, one sees typical $2 \mathrm{D}$ van Hove singularities, and in particular, a well-defined minimum above zero. In the Chern-insulator region of $\Delta / t_{2}$, on the other hand, the density of overlap values shows a tail extending all the way to zero.

In summary, when the system is in its normal-insulator phase, the construction of Boys orbitals for finite samples, or of WFs for periodic samples, can be carried out in the usual way using a projection method. However, once the NI/CI phase boundary has been crossed, such a construction is bound to fail because of singularities that appear in the overlap matrices in both the real-space finite-sample and $\mathbf{k}$-space extended-sample approaches.

\section{THE SPREAD FUNCTIONAL}

Another quantity that shows interesting behavior as the phase boundary is crossed is the spread functional $\Omega$ in real space, defined by Marzari and Vanderbilt $(\mathrm{MV})^{6}$ to be

$$
\Omega=\sum_{n}\left[\left\langle\mathbf{0} n\left|r^{2}\right| \mathbf{0} n\right\rangle-\langle\mathbf{0} n|\mathbf{r}| \mathbf{0} n\rangle^{2}\right],
$$

where $|\mathbf{0} n\rangle$ refers to the WF $|\mathbf{R} n\rangle$ for band $n$ in the home unit cell $\mathbf{R}=\mathbf{0}$ and the sum is over occupied bands of the insulator. The spread functional is a measure of how "spread out" or delocalized the WFs are. In the remainder of this section, we specialize for simplicity to the case of a single band in two dimensions, so that $\Omega=$ $\left\langle\mathbf{0}\left|r^{2}\right| \mathbf{0}\right\rangle-\langle\mathbf{0}|\mathbf{r}| \mathbf{0}\rangle^{2}$. MV showed that the spread functional can be decomposed as $\Omega=\Omega_{I}+\widetilde{\Omega}$, where

$$
\Omega_{I}=\left\langle\mathbf{0}\left|r^{2}\right| \mathbf{0}\right\rangle-\sum_{\mathbf{R}}|\langle\mathbf{0}|\mathbf{r}| \mathbf{R}\rangle|^{2}
$$

and

$$
\widetilde{\Omega}=\sum_{\mathbf{R} \neq \mathbf{0}}|\langle\mathbf{0}|\mathbf{r}| \mathbf{R}\rangle|^{2}
$$

are gauge-invariant and gauge-dependent contributions, respectively. The gauge-invariant part has been shown to be a useful measure for characterizing the system: $\Omega_{I}$ is finite in insulators and diverges in metals. ${ }^{8}$

MV also gave corresponding $\mathbf{k}$-space expressions for the two parts of the functional. Defining the metric tensor $g_{\mu \nu}=\operatorname{Re}\left\langle\partial_{\mu} u_{\mathbf{k}}\left|Q_{\mathbf{k}}\right| \partial_{\nu} u_{\mathbf{k}}\right\rangle$ where $Q_{\mathbf{k}}=1-\left|u_{\mathbf{k}}\right\rangle\left\langle u_{\mathbf{k}}\right|$ (and $\partial_{\mu}=\partial / \partial k_{\mu}$ ), these two quantities can be rewritten as

$$
\Omega_{I}=\frac{\mathcal{A}}{(2 \pi)^{2}} \int_{\mathrm{BZ}} d \mathbf{k} \operatorname{Tr}[g(\mathbf{k})]
$$

and

$$
\widetilde{\Omega}=\frac{\mathcal{A}}{(2 \pi)^{2}} \int_{\mathrm{BZ}} d \mathbf{k}|\mathbf{A}(\mathbf{k})-\overline{\mathbf{A}}|^{2},
$$

where $\mathcal{A}$ is the unit cell area, $\operatorname{Tr}[g]=g_{x x}+g_{y y}$, and $\overline{\mathbf{A}}$ is the BZ average of $\mathbf{A}(\mathbf{k})$ defined just above Eq. (3).

In the case of a Chern insulator, the use of the realspace expressions (14-15) becomes problematic, since well-localized WFs cannot be constructed. Nevertheless, the reciprocal-space expressions (16-17) remain welldefined. It is interesting, then, to see how these quantities behave in a Chern insulator. Do each of these quantities remain finite, or does one or both of them diverge? Also, what is the behavior of these quantities as one approaches the NI/CI phase boundary?

To answer these questions, we have computed the quantities in Eqs. (16-17) using the finite-difference versions of these equations given in Eqs. (34) and (36) of Ref. [6]. For the calculation of the gauge-dependent part $\widetilde{\Omega}$, we have fixed our gauge such that $\left|\psi_{\mathbf{k}}\right\rangle$ is real for all $\mathbf{k}$ on the lower-energy site in the home unit cell. The results are plotted in Fig. 8 for different densities of the $\mathbf{k}$-mesh. It can be seen that $\Omega_{I}$ is finite inside both the normal and Chern-insulator regions. At the critical value of $\left(\Delta / t_{2}\right)_{\mathrm{cr}} \approx 3.67$, however, $\Omega_{I}$ diverges logarithmically with the number of k-points. Furthermore, $\widetilde{\Omega}$ is finite in the normal insulator region, but diverges logarithmically with the number of $\mathbf{k}$-points for Chern insulators. This latter behavior is consistent with the presence of a vortex in the phases of the $\left|w_{\mathbf{k}}\right\rangle$ around point $\mathbf{k}_{a}$, which causes A to diverge as $\left|\mathbf{k}-\mathbf{k}_{a}\right|^{-1}$ and imparts a logarithmic divergence to Eq. (17). It follows that the total spread $\Omega$ is finite in normal insulators and divergent in Chern insulators. Heuristically, it is tempting to associate this 


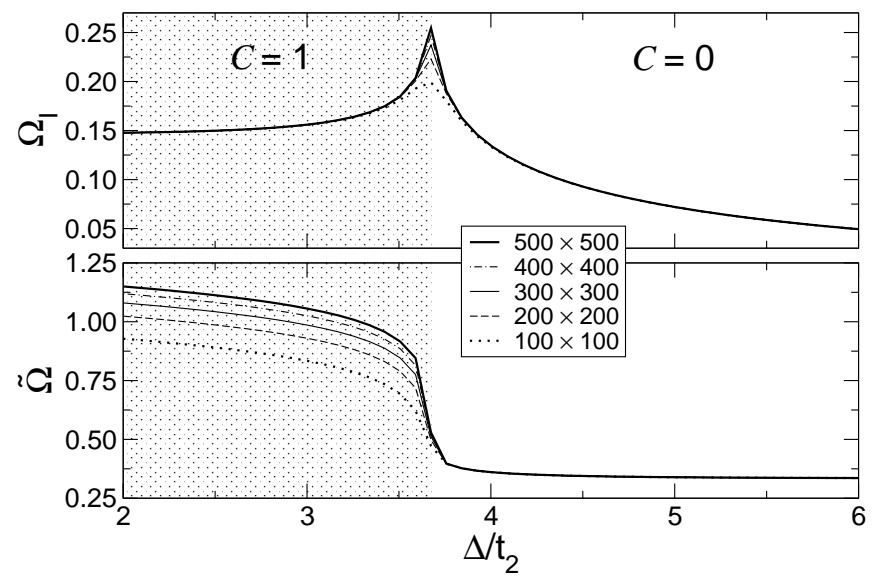

FIG. 8: Gauge-independent part $\Omega_{I}$ and gauge-dependent part $\widetilde{\Omega}$ of the spread functional for the Haldane model as a function of the k-mesh density.

divergence with the presence of the metallic chiral edge states that are required to exist in Chern insulators (see Sec. III), but it is unclear precisely how these features are related. Note that electron localization in the quantum Hall regime is discussed in detail by R. Resta in Ref. [24].

\section{DECAY OF THE DENSITY MATRIX}

The decay of the density matrix is a fundamental property of a system and it is closely connected to the electron localization. It was first studied by W. Kohn for one-dimensional insulators, ${ }^{25}$ and many others have investigated this topic thereafter. ${ }^{7-9,24,26,27}$

For periodic samples the density matrix is defined as

$$
\rho\left(\mathbf{r}, \mathbf{r}^{\prime}\right)=\frac{\mathcal{A}}{(2 \pi)^{2}} \sum_{n=1}^{\text {occ }} \int_{\mathrm{BZ}} d \mathbf{k} \psi_{n \mathbf{k}}^{*}(\mathbf{r}) \psi_{n \mathbf{k}}\left(\mathbf{r}^{\prime}\right)
$$

where we assume that the wave functions $\psi_{n \mathbf{k}}$ are normalized to one unit cell of area $\mathcal{A}$. If the wave functions are written in terms of some basis functions $\phi_{\alpha}^{\mathbf{k}}(\mathbf{r})$,

$$
\psi_{n \mathbf{k}}(\mathbf{r})=\sum_{\alpha} C_{n \alpha}^{\mathbf{k}} \phi_{\alpha}^{\mathbf{k}}(\mathbf{r})
$$

this becomes

$$
\rho\left(\mathbf{r}, \mathbf{r}^{\prime}\right)=\frac{\mathcal{A}}{(2 \pi)^{2}} \sum_{n=1}^{\text {occ }} \sum_{\alpha \beta} \int_{\mathrm{BZ}} d \mathbf{k} C_{n \alpha}^{\mathbf{k} *} C_{n \beta}^{\mathbf{k}} \phi_{\alpha}^{\mathbf{k} *}(\mathbf{r}) \phi_{\beta}^{\mathbf{k}}\left(\mathbf{r}^{\prime}\right) .
$$

The $C_{n \alpha}^{\mathbf{k}}$ are the eigenvectors obtained by diagonalizing the model Hamiltonian - in our case Eq. (4). In a tightbinding model, the basis functions $\phi_{\alpha}^{\mathbf{k}}(\mathbf{r})$ are made up of localized orbitals $\phi$ at sites $\mathbf{r}_{\alpha}$ :

$$
\phi_{\alpha}^{\mathbf{k}}(\mathbf{r})=\sum_{\mathbf{R}} e^{i \mathbf{k} \cdot\left(\mathbf{R}+\mathbf{r}_{\alpha}\right)} \phi\left(\mathbf{r}-\left(\mathbf{R}+\mathbf{r}_{\alpha}\right)\right) .
$$
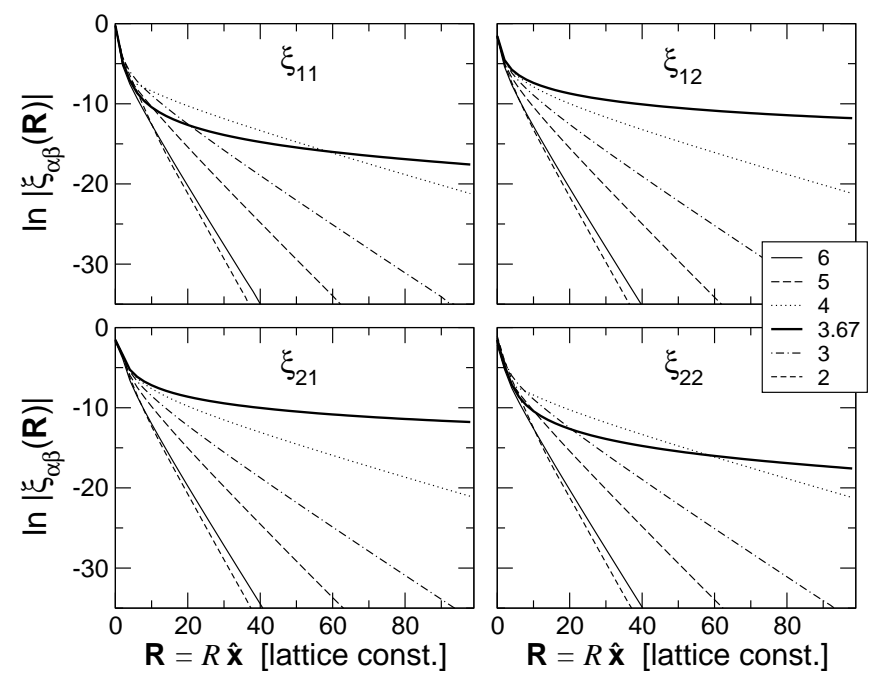

FIG. 9: Logarithm of the density-matrix kernel $\left|\xi_{\alpha \beta}(R \hat{\mathbf{x}})\right|$ of the Haldane model for several values of $\Delta / t_{2}$. The linear asymptotic behavior indicates exponential decay except at $\left(\Delta / t_{2}\right)_{\mathrm{cr}} \approx 3.67$ (solid curves) where the decay is power-law instead.

Inserting Eq. (21) into Eq. (20) gives

$\rho\left(\mathbf{r}, \mathbf{r}^{\prime}\right)=\sum_{\substack{\mathbf{R} \mathbf{R}^{\prime} \\ \alpha \beta}} \xi_{\alpha \beta}\left(\mathbf{R}^{\prime}-\mathbf{R}\right) \phi^{*}\left(\mathbf{r}-\mathbf{R}-\mathbf{r}_{\alpha}\right) \phi\left(\mathbf{r}^{\prime}-\mathbf{R}^{\prime}-\mathbf{r}_{\beta}\right)$

where

$$
\xi_{\alpha \beta}(\mathbf{R})=\frac{\mathcal{A}}{(2 \pi)^{2}} \sum_{n=1}^{\mathrm{occ}} \int_{\mathrm{BZ}} d \mathbf{k} C_{n \alpha}^{\mathbf{k} *} C_{n \beta}^{\mathbf{k}} e^{i \mathbf{k} \cdot\left(\mathbf{R}+\mathbf{r}_{\beta}-\mathbf{r}_{\alpha}\right)} .
$$

The density matrix cannot be evaluated explicitly without the knowledge of the orbitals $\phi$, but we can study instead the decay of $\xi_{\alpha \beta}(\mathbf{R})$, which essentially has the interpretation of being a density matrix expressed in a tight-binding representation.

Calculating the decay of $\xi_{\alpha \beta}(\mathbf{R})$ in Eq. (23) numerically is very demanding and the corresponding results are to be interpreted with caution. To ensure high accuracy, we used a very dense $\mathbf{k}$-mesh of $2000 \times 2000$ points and 128-bit arithmetic. Results for $\xi_{\alpha \beta}(R \hat{\mathbf{x}})$ (i.e., along the $x$ direction) for the Haldane model are collected in Fig. 9. In normal insulators the density matrix decays exponentially with a power-law prefactor. ${ }^{7}$ We therefore choose to fit our results according to $\xi_{\alpha \beta} \sim R^{-a} e^{-b R}$, where $R=|\mathbf{R}|=|R \hat{\mathbf{x}}|$, and $a$ and $b$ are fit parameters. More specifically, we performed least-square fits of $\ln \left|\xi_{\alpha \beta}\right|$ for distances up to 100 unit cells. For the decay behavior at the NI/CI boundary, we even went as far as 500 unit cells.

Within fitting error, the best-fit values for the parameter $b$ are the same for all $\xi_{\alpha \beta}$. Numerical results corresponding to $\Delta / t_{2}$ values of $6,5,4,3.67,3$, and 2 are $0.69 \pm 1,0.43 \pm 1,0.118 \pm 5,0.0001 \pm 1,0.282 \pm 1$, and 
$0.75 \pm 1$, respectively. In general, when approaching the phase boundary from either side, the best-fit value of the parameter $b$ decreases and takes its minimum of zero at $\left(\Delta / t_{2}\right)_{\mathrm{cr}}$. In other words, in the normal and Cherninsulator regions the decay is dominated by the exponential behavior. However, exactly at the phase boundary the exponential decay vanishes $(b=0)$ and a pure powerlaw behavior remains, similar to metals. At $\left(\Delta / t_{2}\right)_{\mathrm{cr}}$ the power-law decay is then characterized by $a=3.01 \pm 3$ for $\xi_{11}$ and $\xi_{22}$, and $a=2.00 \pm 2$ for $\xi_{12}$ and $\xi_{21}$, which suggests that the "true" values are the integers 3 and 2 . Note that the results depicted in Fig. 9 correspond to a particular direction in real space $(\mathbf{R}=R \hat{\mathbf{x}})$. While the decay parameters inside the normal and Chern-insulator phase depend slightly on the direction, they become universal at $\left(\Delta / t_{2}\right)_{\mathrm{cr}}$. Again, this is a signature of the metallic character.

It is interesting that the power of the power-law decay at the phase boundary seems to be exactly an integer and that it differs by 1 for different $\xi_{\alpha \beta}$. This behavior can be understood in the following way: $\xi_{\alpha \beta}(\mathbf{R})$ of Eq. (23) is essentially the Fourier transform of the kernel $C_{n \alpha}^{\mathbf{k} *} C_{n \beta}^{\mathbf{k}}$ and it is well known that discontinuities in the kernel determine the decay behavior of the resulting quantity. In one dimension the discontinuities are related to the decay like $R^{-(l+1)}$, where $l$ is the number of continuous derivatives of the kernel. ${ }^{28,29}$ Unfortunately, in two dimensions the situation is more complex and the resulting BZ integrals cannot easily be solved analytically. Nevertheless, we give heuristic arguments that a similar expression holds for higher dimensions.

To this end, we solve for analytic expressions of $C_{n \alpha}^{\mathbf{k}}$ by diagonalizing the Hamiltonian $H(\mathbf{k})$ in Eq. (4). In turn, we find analytic expressions for the kernel $C_{n \alpha}^{\mathbf{k *}} C_{n \beta}^{\mathbf{k}}$. Next, we switch to polar coordinates $\mathbf{k}=\left(k_{x}, k_{y}\right) \rightarrow \mathbf{k}=(k, \phi)$, replace $\Delta / t_{2}$ by $\left(\Delta / t_{2}\right)_{\mathrm{cr}}=3 \sqrt{3} \sin \varphi$, and expand the kernel around the Dirac point in orders of $k$ :

$$
\begin{aligned}
C_{11}^{\mathbf{k} *} C_{11}^{\mathbf{k}}= & \frac{1}{2}-\frac{3 t_{2} \sin \varphi}{4 t_{1}} k \\
& +\frac{5 t_{2} \cos 3 \phi \sin \varphi}{16 \sqrt{3} t_{1}} k^{2} \\
& +\mathcal{O}\left(k^{3}\right) \\
C_{11}^{\mathbf{k} *} C_{12}^{\mathbf{k}}= & \frac{(1-i \sqrt{3}) e^{-i \phi}}{4} \\
& +\frac{(3+i \sqrt{3}) e^{-i \phi} \sin 3 \phi}{48} k \\
& +\mathcal{O}\left(k^{2}\right)
\end{aligned}
$$

From the above expansions it is apparent that $C_{11}^{\mathbf{k} *} C_{11}^{\mathbf{k}}$ has its first discontinuity in first order in $k$. Hence, there are $l=1$ continuous derivatives. On the other hand, due to the $e^{-i \phi}$ term, $C_{11}^{\mathbf{k} *} C_{12}^{\mathbf{k}}$ has already a discontinuity in zeroth order in $k$, i.e. $l=0$. This is consistent with the numerical results for $\xi_{\alpha \beta}(\mathbf{R})$ in Fig. 9 if we assume that the decay in two dimensions is according to $R^{-(l+2)}$.
Equations (25) and (24) are thus consistent with a decay of $R^{-2}$ and $R^{-3}$, respectively.

In summary, the numerical and analytical arguments are consistent in supporting the conclusion that the diagonal and off-diagonal elements of $\xi_{\alpha \beta}(\mathbf{R})$ decay as $R^{-3}$ and $R^{-2}$ respectively. An arbitrary pair of coordinates $\mathbf{r}$ and $\mathbf{r}^{\prime}$ in Eq. (22) will involve a linear combination of contributions coming from diagonal and off-diagonal terms, so the final conclusion is that the decay of the density matrix will be as $R^{-2}$ exactly on the NI/CI boundary, and exponential for any point lying within the normalinsulator or Chern-insulator phase.

Above, we have evaluated the density matrix $\rho\left(\mathbf{r}, \mathbf{r}^{\prime}\right)$ for periodic samples. For finite samples, we expect a parallel behavior to hold for points $\mathbf{r}$ and $\mathbf{r}^{\prime}$ deep inside the bulk. However, if both points are chosen to be near the surface of a Chern-insulator sample, one may expect that the presence of metallic chiral edge states will induce a power-law decay with the distance between $\mathbf{r}$ and $\mathbf{r}^{\prime}$ as measured along the perimeter. Preliminary calculations on finite samples appear consistent with this picture.

\section{CONCLUSIONS}

We have performed numerical and analytical calculations to study the behavior of several properties of the Haldane model as the system undergoes a transition from the normal-insulator phase to the Chern-insulator phase. We first showed how the usual methods of constructing Wannier functions break down for Chern insulators. We then investigated several quantities related to electron localization. We found that the total spread functional, which is finite in normal insulators, diverges in the case of a Chern insulator. However, when the spread functional is decomposed into its gauge-independent and gaugedependent parts, the former is found to remain finite in a Chern insulator, while only the latter diverges. The localization length of Resta and Sorella, ${ }^{8}$ which is related to the gauge-independent part of the spread functional, thus remains finite. However, the localization length increases and diverges logarithmically as one approaches the NI/CI transition. Similarly, when inspecting the density matrix, we find that it decays exponentially inside both the normal and Chern-insulator phases, but that the decay length increases as the phase boundary is approached, and the behavior crosses over to a power-law decay exactly at the phase boundary.

We thus find that a system that is sitting right on the NI/CI boundary has a kind of semimetallic character similar to that of graphene, in which the valence and conduction bands touch at one (for the Haldane model) or two (for graphene) Dirac points in the BZ. When the system is in the Chern-insulator phase, it still has remnants of metallic behavior in the presence of metallic edge states, the divergence of the total spread functional, and the difficulty of constructing Wannier functions.

Our results were obtained here for a specific realiza- 
tion of a Chern insulator, namely, the Haldane model. While it seems very likely that the localization properties found here will apply to other Chern-insulator systems, it remains to test this hypothesis by carrying out similar studies on other systems. It would also be of considerable interest to extend the current study to three-dimensional Chern-insulator crystals, and to continuum, as opposed to tight-binding, models. These could be fruitful avenues for future investigations.

\section{Acknowledgments}

We acknowledge fruitful discussions with R. Resta and D. Ceresoli. This work was supported by NSF grants No. DMR-0233925 and DMR-0549198.
1 D. J. Thouless, Topological Quantum Numbers in Nonrelativistic Physics (World Scientific, Singapore, 1998).

${ }^{2}$ F. D. M. Haldane, Phys. Rev. Lett. 61, 2015 (1988).

3 R. D. King-Smith and D. Vanderbilt, Phys. Rev. B 47, R1651 (1993); D. Vanderbilt and R. D. King-Smith, Phys. Rev. B 48, 4442 (1993).

4 T. Thonhauser, D. Ceresoli, D. Vanderbilt, and R. Resta, Phys. Rev. Lett. 95, 137205 (2005).

${ }^{5}$ D. Ceresoli, T. Thonhauser, D. Vanderbilt, and R. Resta, Phys. Rev. B 74, 24408 (2006).

${ }^{6}$ N. Marzari and D. Vanderbilt, Phys. Rev. B 56, 12847 (1997).

7 L. He and D. Vanderbilt, Phys. Rev. Lett. 86, 5341 (2001).

8 R. Resta and S. Sorella, Phys. Rev. Lett. 82, 370 (1999).

9 I. Souza, T. Wilkens, and R. M. Martin, Phys. Rev. B 62, 1666 (2000).

${ }^{10}$ R. Resta, J. Phys.: Condens. Matter 14, R625 (2002).

11 Y. Hatsugai, J. Phys. Soc. Japan 74, 1374 (2005).

12 X.-G. Wen, Advances in Physics 44, 405 (1995).

13 X.-G. Wen, Phys. Rev. Lett. 84, 3950 (2000).

14 C. Day, Phys. Today 58, No. 2, 17 (2005).

15 For an interacting system with ground-state degeneracy, the Chern invariant is rationally related to a $\mathbf{G}$-vector.

16 D. J. Thouless, M. Kohmoto, M. P. Nightingale, and M. den Nijs, Phys. Rev. Lett. 49, 405 (1982).

17 G. Panati, unpublished (http://www.arxiv.org/abs/mathph/0601034).

18 M. Wilson, Phys. Today 59, No. 1, 21 (2006).

19 D. N. Sheng, Z. Y. Weng, L. Sheng, and F. D. M. Haldane, Phys. Rev. Lett. 97, 036808 (2006).

20 B. I. Halperin, Phys. Rev. B 25, 2185 (1982).

21 D. Yoshioka, The Quantum Hall Effect (Springer, Berlin, 2002).

22 S. F. Boys, Rev. Mod. Phys. 32, 296 (1960); J. M. Foster and S. F. Boys, ibid. 300.

23 B. C. Carlson and J. M. Keller, Phys. Rev. 105, 102 (1957).

24 R. Resta, Phys. Rev. Lett. 95, 196805 (2005).

25 W. Kohn, Phys. Rev. 133, A171 (1964).

26 S. N. Taraskin, D. A. Drabold, and S. R. Elliott, Phys. Rev. Lett. 88, 196405 (2002).

27 S. N. Taraskin, P. A. Fry, Xiaodong Zhang, D. A. Drabold, and S. R. Elliott, Phys. Rev. B 66, 233101 (2002).

28 S. Goedecker, Rev. Mod. Phys. 71, 1085 (1999).

29 M. J. Lighthill, Introduction to Fourier Analysis and Generalized Functions (Cambridge University Press, Cambridge, 1958). 